\title{
Correspondence
}

\section{Effects of sleep state and feeding on cranial blood flow of the human neonate}

Sir,

We were interested in Rahilly's recent speculation that metabolic changes after feeding may be important factors which could explain the fall in cerebral blood flow he reported one hour after a breast feed in term neonates. ${ }^{1}$ Rahilly went on to imply that the metabolic fluctuations induced by bolus feeding regimens in preterm infants could predispose such infants to intraventricular haemorrhage. The concept that phasic changes in metabolism induced by feeding could induce a variety of cyclical physiological events in neonates is an important and a relatively unresearched subject which may indeed have implications for the nutritional management of sick low birthweight infants. We should like to comment on how far Rahilly's suggestions agree with some of our own data $^{2}$ on the metabolic and endocrine effects of feeding term and preterm infants in the first days after birth.

An argument which links postprandial changes in blood flow to metabolic events presupposes that there are indeed changes in metabolism after a feed. With respect to several circulating metabolites and alimentary hormones, many of which change after a feed in adults, we found that in breast-fed term infants on the 6th day and in preterm infants at means of either 2.5 or 6 days of age, there was no postprandial alteration in ketones (acetoacetate and $\beta$-hydroxybutyrate), alanine, pyruvate, lactate, glucagon, enteroglucagon, vasoactive intestinal peptide, gastrin, secretin, gastric inhibitory polypeptide, or pancreatic polypeptide. In contrast older neonates ( $>3$ weeks, and older than Rahilly's patients), showed pronounced postprandial changes in many of these factors. We do accept however, that the measurement of circulating metabolites may not necessarily reflect the metabolic effects of feeding, which can be assessed fully only by turnover studies.

Nevertheless, phasic metabolic and endocrine changes after a feed in the first week postnatally are not totally absent. Insulin and glucose rose postprandially in all the groups we studied and, perhaps of most interest, the neurotensin response to feeding at this age is pronounced. Neurotensin is one of several gut hormones to have been isolated in both gut and brain. ${ }^{3-4}$ In the brain neurotensin is believed to have a neurotransmitter role, and pharmacological studies in animals suggest that in the circulation this hormone may affect vascular tone. Could the neurotensin response to feeding partly explain Rahilly's findings? Clearly the whole subject needs further study.

\section{References}

1 Rahilly P M. Effects of sleep state and feeding on cranial blood flow of the human neonate. Arch Dis Child 1980; 55: 265-70.

2 Lucas A, Bloom S R, Aynsley-Green A. Development of gut hormone responses to feeding in neonates. Arch Dis Child 1980; 55: 678-82.

3 Brown M, Rivier J, Kobayashi R, Vale W. Neurotensinlike and bombesin-like peptides: CNS distribution and actions. In: Bloom S R, ed. Gut hormones. Edinburgh: Churchill Livingstone, $1978 ; 550-8$.

4 Buchan A M, Polak J M, Sullivan S, Bloom S R, Brown M, Pearse A G E. Neurotensin in the gut. In: Bloom S R, ed. Gut hormones. Edinburgh; Churchill Livingstone, 1978: 544-9.

Alan Lucas

Department of Paediatrics, New Addenbrooke's Hospital, Hills Road, Cambridge CB2 $2 Q Q$

Albert Aynsley-GrefN Department of Paediatrics, John Radcliffe Hospital, Headington, Oxford $O X 39 D U$

\section{Upper airway abnormalities and their functional implications in respiratory disease, especially cot death in infancy}

Sir,

The discussion on the interesting findings reported by Wailoo and Emery ${ }^{1}$ could have been developed further and therefore might have been less speculative. In particular the relationship between tracheal enlargement and function might have been more conclusive if more information had been provided.

A comparison between cot deaths with and without established respiratory disease would have been helpful. It would have clarified any causative role of respiratory disease in tracheal enlargement and the possibility that an enlarged or distensible trachea might predispose to cot death, perhaps by compromising the respiratory response to airways challenge. Wailoo and Emery ${ }^{1}$ referred to previous 'unexplained' findings of vocal cord necrosis in infants dying from cot deaths. As their paper sought a better understanding of abnormal function, surely these two findings could have been compared in the same children. It has been suggested that vocal cord necrosis may be caused by excessive laryngeal adduction, as occurs with the expiratory grunt in respiratory distress. ${ }^{2}$

We now know that expiratory laryngeal adduction often leading to transient glottic closure and thus to 
apposition of the cords can occur normally during quiet sleep in young lambs. ${ }^{3}$ Briefly, this 'braking' of expiratory airflow by the larynx generates positive subglottic pressure of up to $10 \mathrm{~cm}$ water and is an important mechanism in preventing lung collapse in the young until a noncollapsible chest wall is established. If this expiratory laryngeal pressure is prevented, such as by a tracheotomy or endotracheal intubation, respiratory frequency falls and respiratory drive is greatly diminished especially during quiet sleep. Equally, it can be reasoned, if an overdistensible trachea exists or develops, respiratory drive could diminish, and failure or 'unexplained death' occur during respiratory challenge-such as with an apparently trivial infection or upper airways obstruction during sleep. That is to say, positive expiratory pressure could not be developed if the upper airways simply dilated with glottic adduction.

What subglottic pressures are generated in respiratory disease in the young appears to be uncertain. From our studies in early postnatal life we would expect tracheal distension during the expiratory cycle (rather than obstruction) in quiet sleep but such airway collapse could occur in rapid eye movement sleep when upper airway expiratory resistance is slight.

Thus a hypothesis to be tested could be an association between vocal cord necrosis, indicative of chronic laryngeal adduction in expiration, and a distended trachea in these cases. The information from this comprehensive series of necropsies could provide valuable evidence which could point the way to a functional evaluation in such infants possibly averting later morbidity and mortality. Surely a morbid anatomist's dream!

\section{References}

1 Wailoo M, Emery J L. The trachea in children with respiratory diseases including children presenting as cot deaths. Arch Dis Child 1980; 55: 199-203.

2 Osborn G R, Flett R L. Laryngeal dysfunction and the pulmonary syndrome of the newborn. J Clin Pathol 1962; 15: $527-41$.

3 Johnson P. Comparative aspects of the control of breathing during development. In: von Euler C, Lagercrantz $\mathrm{H}$, eds. Central nervous control mechanisms in breathing. Oxford: Pergamon Press, 1979: 337-52.

P JoHNSON Nuffield Institute for Medical Research, Headley Way, Oxon

\section{Professor Emery and Dr Wailoo comment:}

We were very well aware of most of the points raised by Paul Johnson but did not wish to speculate too far beyond our evidence.

The possibility of spasmodic glottic closure being a major factor in unexpected death is one that we have been studying for many years but unfortunately, as with so many things related to cot death, these vocal cord lesions also occur in children who die in hospital from apparently clinically justifiable disease. ${ }^{1}$ It would be possible, and nice, to do the anatomical correlations that Johnson suggests but the nonspecificity of the vocal cord lesions would be likely to lead to an inconclusive result.

Our own interest has progressed from the general statistical conclusions to the possibility that there is a particular area of tracheomalacia at the carina which enables the trachea to 'block off' at this point and that infection is either absent or present at the laryngeal conus.

It would be of interest to us if Paul Johnson could give his findings regarding the presence or absence of laryngeal lesions indicating spasm in his lambs!

\section{Reference}

1 Cullity G J, Emery J L. Ulceration and necrosis of vocal cords in hospital and unexpected child deaths. $J$ Pathol 1975; 115: 27-31.

J L EMERY AND M P WAILOO Department of Histopathology, Children's Hospital, Western Bank, Sheffield S10 3BR

\section{Gluten intolerance, gluten enteropathy, and coeliac disease}

Sir,

I read with interest the article by Dodge. ${ }^{1}$ His enlarged concept of gluten-induced 'disease' is attractive, original, and may be correct, but his proposal for omitting the second of the present three biopsies recommended by the European Society for Paediatric Gastroenterology and Nutrition (ESPGAN) ${ }^{2}$ seems inopportune and open to criticism.

Most coeliac children put on a gluten-free diet will present a normal jejunal mucosa from as soon as a few months up to 1-2 years after introduction of the diet and if they do not one suspects incomplete gluten withdrawal. ${ }^{3}$ Though some biochemical, haematological, or immunological data can be of help in the follow-up of coeliac children as well as in the assessment of the degree of adherence to the gluten-free diet, a normal jejunal mucosa is the most reliable finding. So, omitting the second biopsy not only fails to confirm the second step to a final diagnosis of gluten-induced enteropathy, but also loses a valuable way of assessing the strictness of dietary treatment.

The criteria of Interlaken, ${ }^{2}$ including 'the 2-year rule', may be too rigid, but nevertheless they fit with most coeliac children $(95 \%)^{4}$ and in our experience they contribute to an increased accuracy in the diagnosis of coeliac disease.

Until a more complete knowledge is reached concerning permanent, transient (?), ${ }^{5}$ or latent (?) ${ }^{6}$ gluten enteropathy or gluten intolerance any escape from the ESPGAN criteria seems undesirable and will confuse rather than help to clarify the diagnosis and management of children with coeliac disease. 